\title{
Andronov-Hopf bifurcation and sensitivity analysis of a time-delay HIV model with logistic growth and antiretroviral treatment
}

\author{
Rachadawan Darlai ${ }^{{ }^{*}+}(\mathbb{D}$ ) and Elvin J Moore $2,3 \dagger$
}

\author{
"Correspondence: \\ rachadawan.d@sciee.kmutnb.ac.th \\ ${ }^{1}$ Faculty of Science, Energy and \\ Environment, King Mongkuts \\ University of Technology North \\ Bangkok (Rayong Campus), Rayong, \\ Thailand \\ Full list of author information is \\ available at the end of the article \\ ${ }^{\dagger}$ Equal contributors
}

\begin{abstract}
A mathematical model of the infection of CD4+ T-cells by HIV that includes the effects of treatment by a reverse transcriptase inhibitor (RTI) and a protease inhibitor (PI) is studied. The model includes three populations of CD4+ T-cells (healthy cells, latently-infected cells which cannot produce virus, and productively-infected cells which can produce virus) and two populations of free virus in the blood (infectious virus and non-infectious virus). The model includes a time delay between a T-cell becoming latently infected and productively infected. The model has a virus-free and a chronic infection equilibrium. It is shown that the model has Andronov-Hopf bifurcations leading to limit cycle behavior in the chronic infection region at critical values of the time delays. For three data sets obtained from the work of previous authors, numerical simulations have given critical delay values ranging from approximately 15 days to more than 200 days. This range includes the period of approximately 50 days for intermittent viral blips reported by Rong and Perelson (Plos Comp. Biol. 5(10), 1-18 (2009)). Simple formulas are derived for the sensitivity indices of the equilibrium populations and the basic reproductive number with respect to all parameters in the model. Numerical simulations are carried out to support the analytical results. The numerical results suggest that the most effective methods of reducing both the basic reproductive number and the chronic infection CD4+ T-cell and virus populations are the following: (1) to increase the efficacy of the antiretroviral treatments and (2) to increase virus clearance rate, decrease infection rate, or decrease viral reproduction rate.
\end{abstract}

Keywords: HIV model; RTI and PI treatment; limit cycles; viral blips; sensitivity analysis

\section{Introduction}

The development of antiretroviral therapy using reverse transcriptase inhibitors (RTI) and protease inhibitors (PI) has resulted in a big reduction in the disability associated with HIV and with the rate of progression to AIDS. Although there is evidence that antiretroviral therapy does not completely eliminate the virus (see, e.g., [2, 3]), there is recent evidence that antiretroviral therapy can reduce the level of virus in an HIV person below detectable levels (see, e.g., $[1,4-6]$ ) and that it can depress the HIV level in an HIV+ person sufficiently to effectively stop transmission of HIV from an HIV+ person to an uninfected person (see, e.g., [7-9]). However, in many countries antiretroviral therapy is not avail-

(c) The Author(s) 2017. This article is distributed under the terms of the Creative Commons Attribution 4.0 International License (http://creativecommons.org/licenses/by/4.0/), which permits unrestricted use, distribution, and reproduction in any medium, provided you give appropriate credit to the original author(s) and the source, provide a link to the Creative Commons license, and indicate if changes were made. 
able. Also, infection by HIV can be asymptomatic [3], and these asymptomatic infected people may interact normally with people and pass on the disease to uninfected people.

Many researchers have developed mathematical models in an attempt to develop an understanding of HIV transmission at either the cell level (see, e.g., $[1,5,6,10-15]$ ) or the population level (see, e.g., $[16,17]$ ).

In this paper, we consider a model for HIV infection at the cell level recently discussed by Wang et al. [15]. The model includes three populations of CD4+ T-cells (healthy cells, latently-infected cells which cannot produce virus, and productively-infected cells which can produce virus) and two populations of free virus (infectious virus and non-infectious virus). The model also includes the effects of treatments with a reverse transcriptase inhibitor (RTI) and a protease inhibitor (PI). In their paper, Wang et al. showed that the model has virus-free and chronic infection equilibrium solutions and they proved local and global stability, boundedness and positivity of these solutions. They also used a latin hypercube sampling technique for sensitivity analysis of the parameters in their model.

The model of Wang et al. is based on a model discussed by Rong and Perelson $[1,5,6]$ with the main difference being the addition of a logistic growth term for healthy CD4+ T-cells. One of the important questions discussed in the Rong and Perelson papers is the mechanism that produces an intermittent viral blip with a period of approximately 50 days when subjects are treated with highly active antiretroviral therapy (HAARV). It is well known (see, e.g., [18]) that time delays can produce bifurcations leading to limit cycle behavior in both discrete-time and continuous-time dynamical systems. One of the aims of the present paper is to check if time delays for procession of latently infected T-cells to productively infected T-cells could result in viral blip-type behavior.

In the present paper, we develop simple analytical formulas for sensitivity indices for the basic reproductive number, and for the virus-free and chronic equilibrium populations of the time-delay model. We also show that the time-delay model can undergo AndronovHopf bifurcations in the chronic equilibrium solutions at critical time delays and that limit cycle behavior in all five populations occurs at time delays greater than the critical values.

\section{Time-delay model}

We consider the following model, which is generalized from the model of Wang et al. [15] by including a time delay for the procession of latently infected T-cells to productively infected T-cells. The variables in the model are defined in Table 1, and the parameters are defined in Table 2.

$$
\begin{aligned}
& \frac{d T(t)}{d t}=\Lambda-d_{T} T(t)+r T(t)\left(1-\frac{T(t)}{T_{\max }}\right)-\left(1-n_{r t}\right) k V(t) T(t), \\
& \frac{d L(t)}{d t}=\eta\left(1-n_{r t}\right) k V(t) T(t)-d_{L} L(t)-a L(t-\tau), \\
& \frac{d I(t)}{d t}=(1-\eta)\left(1-n_{r t}\right) k V(t) T(t)-d_{I} I(t)+a L(t-\tau), \\
& \frac{d V(t)}{d t}=\left(1-n_{p}\right) N d_{I} I(t)-c V(t), \\
& \frac{d W(t)}{d t}=n_{p} N d_{I} I(t)-c W(t) .
\end{aligned}
$$


Table 1 Variables in the model (adapted from $[1,5,6,15]$ )

\begin{tabular}{ll}
\hline Population & Definition \\
\hline$T(t)$ & Healthy CD4+T-cells at time $t$ \\
$L(t)$ & Latently infected CD4+T-cells at time $t$ \\
$I(t)$ & Productively infected CD4+T-cells at time $t$ \\
$V(t)$ & Free infectious virus at time $t$ \\
$W(t)$ & Free non-infectious (inhibited) virus at time $t$ \\
\hline
\end{tabular}

Table 2 Parameters in the model (adapted from $[1,5,6,15]$ )

\begin{tabular}{ll}
\hline Parameter & Definition \\
\hline$\Lambda$ & Constant production rate of healthy CD4+ T-cells from precursors \\
$d_{T}$ & Natural death rate of healthy CD4+ T-cells \\
$r$ & Logistic growth rate of healthy CD4+ T-cells \\
$T_{\max }$ & Carrying capacity of healthy CD4+ T-cells \\
$k$ & Infection rate of healthy CD4+ T-cells by free infectious virus \\
$\eta$ & Fraction of infections leading to latently infected CD4+ T-cells \\
$d_{L}$ & Death rate of latently infected CD4+ T-cells \\
$d_{l}$ & Death rate of productively infected CD4+ T-cells \\
$a$ & Activation rate from latently to productively infected CD4+ T-cells \\
$N$ & Average number of free virus released by a productively infected CD4+ T-cell during its mean \\
$c$ & lifetime 1/d, \\
$n_{r t}$ & Clearance rate of free virus \\
$n_{p}$ & Drug efficacy of RTI $\left(0 \leq n_{r t}<1\right)$ \\
$\tau$ & Drug efficacy of PI $\left(0 \leq n_{p}<1\right)$ \\
\hline
\end{tabular}

\section{Equilibrium points}

As shown by Wang et al. [15] the system (1)-(5) has a virus-free and a chronic equilibrium point. For completeness, we will briefly summarize the proof here.

Theorem 1 The system (1)-(5) has two equilibrium points:

1. Virus-free:

$$
\left(T_{1}^{*}, L_{1}^{*}, I_{1}^{*}, V_{1}^{*}, W_{1}^{*}\right)=\left(\frac{T_{\max }}{2 r}\left(r-d_{T}+\sqrt{\left(r-d_{T}\right)^{2}+4 \frac{r \Lambda}{T_{\max }}}\right), 0,0,0,0\right) .
$$

2. Chronic infection:

$$
\begin{aligned}
& T_{2}^{*}=\frac{c\left(a+d_{L}\right)}{\bar{k} \bar{N}\left(a+(1-\eta) d_{L}\right)}, \quad L_{2}^{*}=\frac{c \eta}{\bar{N}\left(a+(1-\eta) d_{L}\right)} V_{2}^{*}, \\
& I_{2}^{*}=\frac{c}{\bar{N} d_{I}} V_{2}^{*}, \quad V_{2}^{*}=\frac{1}{\bar{k}}\left(\frac{T_{1}^{*}}{T_{2}^{*}}-1\right)\left(\frac{\Lambda}{T_{1}^{*}}+r \frac{T_{2}^{*}}{T_{\max }}\right), \\
& W_{2}^{*}=\frac{n_{p} N}{\bar{N}} V_{2}^{*},
\end{aligned}
$$

where $\bar{k}=\left(1-n_{r t}\right) k, \bar{N}=\left(1-n_{p}\right) N$.

\section{Proof}

1. Virus-free equilibrium. Setting $L_{1}^{*}=I_{1}^{*}=V_{1}^{*}=W_{1}^{*}=0$, we have $\frac{d L}{d t}=\frac{d I}{d t}=\frac{d V}{d t}=\frac{d W}{d t}=0$. Then, setting $\frac{d T}{d t}=0$ and choosing the positive solution for $T_{1}^{*}$ gives the result in part 1 of the theorem. 
2. Chronic equilibrium. The equations for $I_{2}^{*}$ and $W_{2}^{*}$ follow immediately from the conditions $\frac{d V}{d t}=\frac{d W}{d t}=0$. Then, setting $\frac{d L}{d t}=0$ gives

$$
L_{2}^{*}=\frac{\eta}{a+d_{L}} \bar{k} V_{2}^{*} T_{2}^{*}
$$

Then, adding equations (2) and (3), setting $\frac{d(L+I)}{d t}=0$, and substituting in equation (8), we obtain the equation for $T_{2}^{*}$ in (7). Then, substituting for $T_{2}^{*}$ in (8), we obtain the equation for $L_{2}^{*}$ in (7). Finally, setting $\frac{d T}{d t}=0$, we obtain

$$
\begin{aligned}
V_{2}^{*} & =\frac{1}{\bar{k} T_{2}^{*}}\left(\Lambda+T_{2}^{*}\left(r-d_{T}-r \frac{T_{2}^{*}}{T_{\max }}\right)\right) \\
& =\frac{1}{\bar{k}}\left(\frac{T_{1}^{*}}{T_{2}^{*}}-1\right)\left(\frac{\Lambda}{T_{1}^{*}}+r \frac{T_{2}^{*}}{T_{\max }}\right) .
\end{aligned}
$$

The proof is complete.

Note: The chronic infected virus population $V_{2}^{*}$ is greater than 0 , i.e., the chronic equilibrium exists, if and only if the parameter $R_{0}=\frac{T_{1}^{*}}{T_{2}^{*}}>1$. We shall show in the next section that $R_{0}$ is the basic reproductive number of the model.

\section{Basic reproductive number}

For this model, as in most models with a virus-free and a chronic equilibrium state, there are three methods of determining the basic reproductive number. They are: (1) Lyapunov's first method of checking the eigenvalues of the Jacobian of the linearized system at an equilibrium point (see, e.g., [19]), (2) the next-generation method of van den Driessche and Watmough [20], or (3) by finding the condition for the existence of the chronic equilibrium as in equation (9).

\subsection{Next-generation method}

In using the next-generation method, it is necessary to identify a suitable infected population in the model to choose as an initial infected population. Possible populations are $L, I, V$. In this model $L$ and $I$ cannot be considered as separate initially infected populations since both infections come from infection of the susceptible population $T$ by contact with the $V$ population. The population $V$ comes only from one population (the $I$ population), and therefore we choose it as the initial infected population for the next-generation method and order the variables as $[V, T, L, I, W]^{T}$. As stated in Wang et al. [15], the nextgeneration method gives

$$
R_{0}=\frac{a+(1-\eta) d_{L}}{c\left(a+d_{L}\right)} \overline{k N} T_{1}^{*}=\frac{T_{1}^{*}}{T_{2}^{*}}
$$

This formula for $R_{0}$ is in agreement with the result derived in (9) as the condition for existence of the chronic equilibrium.

\subsection{Linearized equations and stability}

For the time-delay model (1)-(5), the linearized equations about an equilibrium point are (we let $T(t)=T^{*}+x_{1}(t), L(t)=L^{*}+x_{2}(t), I(t)=I^{*}+x_{3}(t), V(t)=V^{*}+x_{4}(t)$, and $W(t)=$ 
$W^{*}+x_{5}(t)$, where $x_{j}(T)$ are small perturbations)

$$
\begin{aligned}
& \frac{d x_{1}(t)}{d t}=-d_{T} x_{1}(t)+r x_{1}(t)\left(1-2 \frac{T^{*}}{T_{\max }}\right)-\bar{k} V^{*} x_{1}(t)-\bar{k} x_{4}(t) T^{*}, \\
& \frac{d x_{2}(t)}{d t}=\eta \bar{k} V^{*} x_{1}(t)+\eta \bar{k} x_{4}(t) T^{*}-d_{L} x_{2}(t)-a x_{2}(t-\tau), \\
& \frac{d x_{3}(t)}{d t}=(1-\eta) \bar{k} x_{4}(t) T^{*}+(1-\eta) \bar{k} x_{1}(t) V^{*}-d_{I} x_{3}(t)+a x_{2}(t-\tau), \\
& \frac{d x_{4}(t)}{d t}=\bar{N} d_{I} x_{3}(t)-c x_{4}(t), \\
& \frac{d x_{5}(t)}{d t}=n_{p} N d_{I} x_{3}(t)-c x_{5}(t) .
\end{aligned}
$$

Equation (11) can be written in matrix form as $\frac{d x}{d t}=J x(t)$, where $x=\left(x_{1}, x_{2}, x_{3}, x_{4}, x_{5}\right)^{T}$ and $J$ is a Jacobian. Then, assuming a trial solution of the standard form $x(t)=e^{\lambda t} v$, where $v$ is a constant vector, we obtain the Jacobian

$$
J=\left[\begin{array}{ccccc}
-d_{T}+r\left(1-2 \frac{T^{*}}{T_{\max }}\right)-\bar{k} V^{*} & 0 & 0 & -\bar{k} T^{*} & 0 \\
\eta \bar{k} V^{*} \bar{k} \bar{k} V^{*} & -d_{L}-a e^{-\lambda \tau} & 0 & \eta \bar{k} T^{*} & 0 \\
(1-\eta) \bar{k} V^{-\lambda \tau} & -d_{I} & (1-\eta) \bar{k} T^{*} & 0 \\
0 & 0 & \bar{N} d_{I} & -c & 0 \\
0 & 0 & n_{p} N d_{I} & 0 & -c
\end{array}\right]
$$

Using the Routh-Hurwitz conditions (see, e.g., [19]), Wang et al. [15] showed for the case of zero time delay that the eigenvalues of the Jacobian for the virus-free equilibrium have negative real parts for $R_{0}=\frac{T_{1}^{*}}{T_{2}^{*}}<1$ and the eigenvalues of the Jacobian for the chronic equilibrium have negative real parts for $R_{0}>1$. In a later section, we will use the Jacobian to find the critical time delays for Andronov-Hopf bifurcations.

Note: With modern mathematical software such as Matlab, Maple or Mathematica, it is, of course, very easy to numerically compute the eigenvalues of the Jacobian for both the virus-free and chronic equilibrium points for the case of zero time delay.

\section{Sensitivity indices}

We define normalized sensitivity indices for a quantity $Q$ with respect to a parameter $h$ as

$$
S I(Q \mid h)=\frac{h}{Q} \frac{\partial Q}{\partial h}=h \frac{\partial}{\partial h} \ln (Q) .
$$

There are at least three possible methods of computing sensitivity indices: (1) direct computation by differentiation of formulas for the quantity $Q$, (2) the method of Chitnis et al. [21] of linearizing the original nonlinear model equations to set up a system of linear algebraic equations for the sensitivity indices and then numerically solve these equations, and (3) the method used in Wang et al. [15] based on a Latin hypercube sampling technique.

In this paper, we use the first method of direct differentiation as it gives explicit formulas for the indices. We will first compute the sensitivity indices for $T_{1}^{*}, T_{2}^{*}$ with respect to the 
Table 3 Sensitivity indices for virus-free healthy T-cell population $T_{1}^{*}$

\begin{tabular}{llll}
\hline Parameter & Index & Parameter & Index \\
\hline$T_{\max }$ & $1-\frac{2 r \Lambda}{T_{\max } D}$ & $\Lambda$ & $\frac{2 r}{T_{\max } D}$ \\
$r$ & $-\frac{2 r\left(\Lambda-d_{T} T_{1}^{*}\right)}{T_{\max } D}$ & $d_{T}$ & $-\frac{d_{T}}{\sqrt{\left(r-d_{T}\right)^{2}+\frac{4 r \Lambda}{T_{\max }}}}$ \\
\hline
\end{tabular}

Where $D=\left(r-d_{T}\right)^{2}+\frac{4 r \Lambda}{T_{\max }}+\left(r-d_{T}\right) \sqrt{\left(r-d_{T}\right)^{2}+\frac{4 r \Lambda}{T_{\max }}}$.

Table 4 Sensitivity indices for chronic healthy T-cell population $T_{2}^{*}$

\begin{tabular}{llll}
\hline Parameter & Index & Parameter & Index \\
\hline$d_{L}$ & $\frac{a \eta d_{L}}{\left(a+d_{L}\right)\left(a+(1-\eta) d_{L}\right)}$ & $a$ & $-\frac{a \eta d_{L}}{\left(a+d_{L}\right)\left(a+(1-\eta) d_{L}\right)}$ \\
$c$ & 1 & $k$ & -1 \\
$N$ & -1 & $n_{r t}$ & $\frac{n_{r t}}{1-n_{r t}}$ \\
$n_{p}$ & $\frac{n_{p}}{1-n_{p}}$ & $\eta$ & $\frac{\eta d_{L}}{a+(1-\eta) d_{L}}$ \\
\hline
\end{tabular}

parameters by using the formulas in Theorem 1 . We will then compute sensitivity indices for $R_{0}$ using the formula in (10). Finally, we will compute the sensitivity indices for $V_{2}^{*}, L_{2}^{*}$, $I_{2}^{*}$ and $W_{2}^{*}$ using the formulas in Theorem 1 .

\subsection{Sensitivity indices for virus-free healthy T-cell population $T_{1}^{*}$}

From (6), we have

$$
\ln \left(T_{1}^{*}\right)=\ln \left(T_{\max }\right)+\ln \left(r-d_{T}+\sqrt{\left(r-d_{T}\right)^{2}+\frac{4 r \Lambda}{T_{\max }}}\right)-\ln (2)-\ln (r) .
$$

The virus-free equilibrium T-cell population $T_{1}^{*}$ is a function of the four parameters $T_{\max }$, $\Lambda, r$ and $d_{T}$. By differentiation of (14) with respect to these four parameters, we obtain the sensitivity indices shown in Table 3.

\subsection{Sensitivity indices for chronic healthy T-cell population $T_{2}^{*}$}

From Theorem 1, we have

$$
\begin{aligned}
\ln \left(T_{2}^{*}\right)= & \ln (c)+\ln \left(a+d_{L}\right)-\ln \left(1-n_{r t}\right)-\ln (k) \\
& -\ln \left(1-n_{p}\right)-\ln (N)-\ln \left(a+(1-\eta) d_{L}\right) .
\end{aligned}
$$

The chronic equilibrium T-cell population is a function of eight parameters, $d_{L}, a, c, k, N$, $n_{r t}, n_{p}$ and $\eta$. The sensitivity indices of $T_{2}^{*}$ with respect to these parameters are shown in Table 4 .

\subsection{Sensitivity indices for the basic reproductive number $\boldsymbol{R}_{0}$}

From equation (10), we have $\ln \left(R_{0}\right)=\ln \left(T_{1}^{*}\right)-\ln \left(T_{2}^{*}\right)$. We note that $T_{1}^{*}$ and $T_{2}^{*}$ are functions of different sets of parameters. The sensitivity indices are shown in Table 5. 
Table 5 Sensitivity indices for the basic reproductive number $R_{0}$

\begin{tabular}{llll}
\hline Parameter & Index & Parameter & Index \\
\hline$T_{\max }$ & $S I\left(T_{1}^{*} \mid T_{\max }\right)$ & $\Lambda$ & $S I\left(T_{1}^{*} \mid \Lambda\right)$ \\
$r$ & $S I\left(T_{1}^{*} \mid r\right)$ & $d_{T}$ & $S I\left(T_{1}^{*} \mid d_{T}\right)$ \\
$d_{L}$ & $-S I\left(T_{2}^{*} \mid d_{L}\right)$ & $a$ & $-S I\left(T_{2}^{*} \mid a\right)$ \\
$c$ & $-S I\left(T_{2}^{*} \mid C\right)$ & $k$ & $-S I\left(T_{2}^{*} \mid k\right)$ \\
$N$ & $-S I\left(T_{2}^{*} \mid N\right)$ & $n_{r t}$ & $-S I\left(T_{2}^{*} \mid n_{r t}\right)$ \\
$n_{p}$ & $-S I\left(T_{2}^{*} \mid n_{p}\right)$ & $\eta$ & $-S I\left(T_{2}^{*} \mid \eta\right)$ \\
\hline
\end{tabular}

Table 6 Sensitivity indices for chronic productive virus population $V_{2}^{*}$

\begin{tabular}{|c|c|}
\hline Parameter & Index \\
\hline$T_{\max }$ & $\frac{R_{0} S /\left(T_{1}^{*} \mid T_{\max }\right)}{R_{0}-1}-\frac{\Lambda T_{\max } S /\left(T_{1}^{*} \mid T_{\max }\right)+r T_{1}^{*} T_{2}^{*}}{\Lambda T_{\max }+r T_{1}^{*} T_{2}^{*}}$ \\
\hline$\Lambda$ & $\frac{R_{0} S I\left(T_{1}^{*} \mid \Lambda\right)}{R_{0}-1}+\frac{\Lambda T_{\max }\left(1-S I\left(T_{1}^{*} \mid \Lambda\right)\right)}{\Lambda T_{\max }+r T_{1}^{*} T_{2}^{*}}$ \\
\hline r & $\frac{R_{0} S I\left(T_{1}^{*} \mid r\right)}{R_{0}-1}+\frac{r T_{1}^{*} T_{2}^{*}-\Lambda T_{\max } S /\left(T_{1}^{*} \mid r\right)}{\Lambda T_{\max }+r T_{1}^{*} T_{2}^{*}}$ \\
\hline$d_{T}$ & $\frac{R_{0} S I\left(T_{1}^{*} \mid d_{T}\right)}{R_{0}-1}-\frac{\Lambda T_{\max } S I\left(T_{1}^{*} \mid d_{T}^{2}\right)}{\Lambda T_{\max }+r T_{1}^{*} T_{2}^{*}}$ \\
\hline$d_{L}$ & $-\frac{R_{0} S /\left(T_{2}^{*} \mid d_{L}\right)}{R_{0}-1}+\frac{r T_{1}^{*} T_{2}^{*} S I\left(T_{2}^{*} \mid d_{L}\right)}{\Lambda T_{\max }+r T_{1}^{*} T_{2}^{*}}$ \\
\hline$a$ & $\begin{array}{l}-\frac{R_{0} S /\left(T_{2}^{*} \mid a\right)}{R_{0}-1}+\frac{r T_{1}^{*} T_{2}^{*} S /\left(T_{2}^{*} \mid a\right)}{\Lambda T_{\max } r T_{1}^{*} T_{2}^{*}} \\
R_{0} S /\left(T_{2}^{*} \mid c\right) \\
r T_{1}^{*} T_{2}^{*} S /\left(T_{2}^{*} \mid c\right)\end{array}$ \\
\hline C & $\begin{array}{l}R_{0}-1 \\
R_{0} S /\left(T_{2}^{*} \mid k\right)\end{array}$ \\
\hline k & 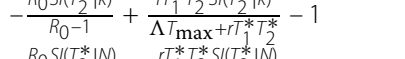 \\
\hline$N$ & $\begin{array}{l}-\frac{R_{0} S /\left(T_{2}^{*} \mid N\right)}{R_{0}-1}+\frac{r T_{1}^{*} T_{2}^{*} S /\left(T_{2}^{*} \mid N\right)}{\Lambda T_{\max }+r T_{1}^{*} T_{2}^{*}} \\
R_{0} S /\left(T_{2}^{*} \mid n_{r t}\right) \\
r T_{1}^{*} T_{2}^{*} S /\left(T_{2}^{*} \mid n_{r t}\right)\end{array}$ \\
\hline$n_{r t}$ & $-\frac{n_{0}}{R_{0}-1}+\frac{1 T^{2}}{\Lambda T_{\max }+r T_{1}^{*} T_{2}^{*}}+\frac{n_{r t}}{1-n_{r t}}$ \\
\hline$n_{p}$ & 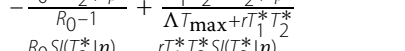 \\
\hline$\eta$ & $-\frac{R_{0} S /\left(I_{2}^{*} \mid \eta\right)}{R_{0}-1}+\frac{r 1_{1}^{*} T_{2}^{*} S /\left(T_{2}^{*} \mid \eta\right)}{\Lambda T_{\max }+r T_{1}^{*} T_{2}^{*}}$ \\
\hline
\end{tabular}

\subsection{Sensitivity indices for the chronic productive virus population $V_{2}^{*}$}

Using the formula for $V_{2}^{*}$ from Theorem 1 , we have

$$
\begin{aligned}
\ln \left(V_{2}^{*}\right)= & \ln \left(R_{0}-1\right)+\ln \left(\frac{\Lambda}{T_{1}^{*}}+\frac{r T_{2}^{*}}{T_{\max }}\right)-\ln \left(1-n_{r t}\right)-\ln (k) \\
= & \ln \left(R_{0}-1\right)+\ln \left(\Lambda T_{\max }+r T_{1}^{*} T_{2}^{*}\right)-\ln \left(T_{\max }\right)-\ln \left(T_{1}^{*}\right) \\
& -\ln \left(1-n_{r t}\right)-\ln (k) .
\end{aligned}
$$

The formulas for the sensitivity indices can then be written in the form given in Table 6 .

\subsection{Sensitivity indices for the chronic infected T-cell populations $L_{2}^{*}, l_{2}^{*}$ and nonproductive virus population $W_{2}^{*}$}

Using the formulas for $L_{2}^{*}, I_{2}^{*}, W_{2}^{*}$ from Theorem 1 , we have

$$
\begin{aligned}
& \ln \left(L_{2}^{*}\right)=\ln (c)+\ln (\eta)+\ln \left(V_{2}^{*}\right)-\ln \left(1-n_{p}\right)-\ln (N)-\ln \left(a+(1-\eta) d_{L}\right) \\
& \ln \left(I_{2}^{*}\right)=\ln (c)+\ln \left(V_{2}^{*}\right)-\ln \left(1-n_{p}\right)-\ln (N)-\ln \left(d_{I}\right) \\
& \ln \left(W_{2}^{*}\right)=\ln \left(n_{p}\right)+\ln \left(V_{2}^{*}\right)-\ln \left(1-n_{p}\right)
\end{aligned}
$$


Table 7 Sensitivity indices for chronic latently infected T-cell population $L_{2}^{*}$

\begin{tabular}{llll}
\hline Parameter & Index & Parameter & Index \\
\hline$T_{\max }$ & $S /\left(V_{2}^{*} \mid T_{\max }\right)$ & $\Lambda$ & $S /\left(V_{2}^{*} \mid \Lambda\right)$ \\
$r$ & $S /\left(V_{2}^{*} \mid r\right)$ & $d_{T}$ & $S /\left(V_{2}^{*} \mid d_{T}\right)$ \\
$d_{L}$ & $S /\left(V_{2}^{*} \mid d_{L}\right)-\frac{(1-\eta) d_{L}}{a+(1-\eta) d_{L}}$ & $a$ & $S /\left(V_{2}^{*} \mid a\right)-\frac{a}{a+(1-\eta) d_{L}}$ \\
$c$ & $1+S /\left(V_{2}^{*} \mid c\right)$ & $k$ & $S /\left(V_{2}^{*} \mid k\right)$ \\
$N$ & $S /\left(V_{2}^{*} \mid N\right)-1$ & $n_{r t}$ & $S /\left(V_{2}^{*} \mid n_{r t}\right)$ \\
$n_{p}$ & $S /\left(V_{2}^{*} \mid n_{p}\right)+\frac{n_{p}}{1-n_{p}}$ & $\eta$ & $1+S I\left(V_{2}^{*} \mid \eta\right)+\frac{\eta d_{L}}{a+(1-\eta) d_{L}}$ \\
\hline
\end{tabular}

Table 8 Sensitivity indices for chronic productively infected T-cell population $I_{2}^{*}$

\begin{tabular}{llll}
\hline Parameter & Index & Parameter & Index \\
\hline$T_{\max }$ & $S /\left(V_{2}^{*} \mid T_{\max }\right)$ & $\Lambda$ & $S /\left(V_{2}^{*} \mid \Lambda\right)$ \\
$r$ & $S /\left(V_{2}^{*} \mid r\right)$ & $d_{T}$ & $S /\left(V_{2}^{*} \mid d_{T}\right)$ \\
$d_{L}$ & $S /\left(V_{2}^{*} \mid d_{L}\right)$ & $d_{l}$ & -1 \\
$a$ & $S I\left(V_{2}^{*} \mid a\right)$ & $c$ & $1+S /\left(V_{2}^{*} \mid c\right)$ \\
$k$ & $S /\left(V_{2}^{*} \mid k\right)$ & $N$ & $S /\left(V_{2}^{*} \mid N\right)-1$ \\
$n_{r t}$ & $S /\left(V_{2}^{*} \mid n_{r t}\right)$ & $n_{p}$ & $S I\left(V_{2}^{*} \mid n_{p}\right)+\frac{n_{p}}{1-n_{p}}$ \\
$\eta$ & $1+S I\left(V_{2}^{*} \mid \eta\right)$ & & \\
\hline
\end{tabular}

Table 9 Sensitivity indices for chronic non-infected free virus population $W_{2}^{*}$

\begin{tabular}{llll}
\hline Parameter & Index & Parameter & Index \\
\hline$T_{\max }$ & $S /\left(V_{2}^{*} \mid T_{\max }\right)$ & $\Lambda$ & $S /\left(V_{2}^{*} \mid \Lambda\right)$ \\
$r$ & $S /\left(V_{2}^{*} \mid r\right)$ & $d_{T}$ & $S /\left(V_{2}^{*} \mid d_{T}\right)$ \\
$d_{L}$ & $S /\left(V_{2}^{*} \mid d_{L}\right)$ & $d_{l}$ & 0 \\
$a$ & $S /\left(V_{2}^{*} \mid a\right)$ & $c$ & $1+S /\left(V_{2}^{*} \mid c\right)$ \\
$k$ & $S /\left(V_{2}^{*} \mid k\right)$ & $N$ & $S /\left(V_{2}^{*} \mid N\right)$ \\
$n_{r t}$ & $S /\left(V_{2}^{*} \mid n_{r t}\right)$ & $n_{p}$ & $S /\left(V_{2}^{*} \mid n_{p}\right)+\frac{1}{1-n_{p}}$ \\
$\eta$ & $S /\left(V_{2}^{*} \mid \eta\right)$ & & \\
\hline
\end{tabular}

The sensitivity indices for $L_{2}^{*}, I_{2}^{*}, W_{2}^{*}$ are shown in Tables 7, 8, 9.

\section{Numerical results}

We give examples of numerical results using the parameter values listed in Table 10 selected from the work of previous authors.

\subsection{Dependence of $\boldsymbol{R}_{0}$ on antiretroviral therapy}

Figures 1(a), (b) and (c) show critical values of the antiretroviral parameters $n_{r t}$ and $n_{p}$ separating the virus-free and chronic infection equilibrium regions $\left(R_{0}=1\right)$ for data sets 1, 2 and 3 of Table 10, respectively. For the three sets it can be seen that the model (1)-(5) predicts that for sufficiently high antiretroviral therapy the HIV infection levels can be reduced to zero. However, as there is evidence (see, e.g., $[2,3]$ ) that the virus cannot be completely eliminated from an HIV+ person, these results suggest that the model requires adjusting in the region of high levels of antiretroviral therapy. 
Table 10 Parameter values (adapted from Wang et al. [15])

\begin{tabular}{|c|c|c|c|c|c|}
\hline Parameter & Set 1 & Set 2 & Set 3 & Unit & Source \\
\hline$T_{\max }$ & 1500 & 1500 & 1500 & $\left.\mu\right|^{-1}$ & {$[14,15]$} \\
\hline$\Lambda$ & 10 & 10 & 10 & $\left.\mu\right|^{-1}$ day $^{-1}$ & {$[1,5,6,14,15]$} \\
\hline$r$ & 0.1 & 0.03 & 0.1 & day $^{-1}$ & {$[14,15]$} \\
\hline$d_{T}$ & 0.03 & 0.01 & 0.01 & day $^{-1}$ & {$[1,5,6,14,15]$} \\
\hline$d_{L}$ & 0.001 & 0.004 & 0.2 & day $^{-1}$ & {$[1,5,6,15]$} \\
\hline$a$ & 0.1 & 0.01 & 0.3 & day $^{-1}$ & {$[1,5,6,15]$} \\
\hline c & 20 & 3 & 15 & day $^{-1}$ & {$[1,5,6,14,15]$} \\
\hline k & 0.0001 & 0.0001 & 0.0001 & $\left.\mu\right|^{-1}$ day $^{-1}$ & {$[1,5,6,14,15,22]$} \\
\hline N & 1000 & 200 & 500 & virions/cell & {$[1,5,6,14,15,22]$} \\
\hline$\eta$ & 0.02 & 0.001 & 0.5 & & {$[1,5,6,15]$} \\
\hline$d_{l}$ & 1 & 1 & 0.8 & day $^{-1}$ & {$[1,5,6,14,15]$} \\
\hline
\end{tabular}

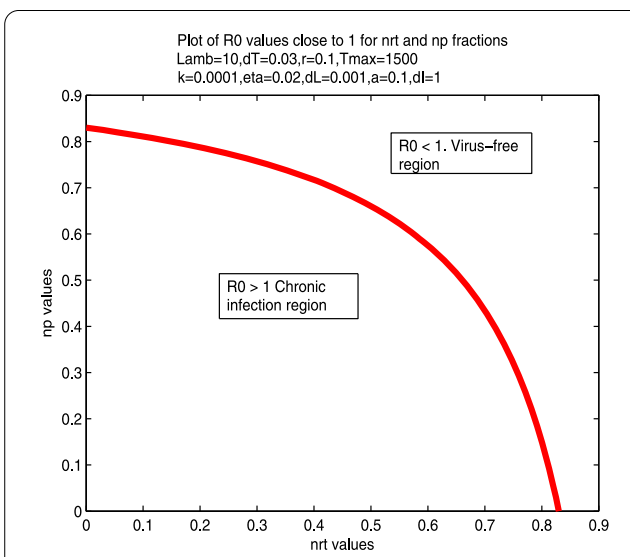

(a) Data set 1 .

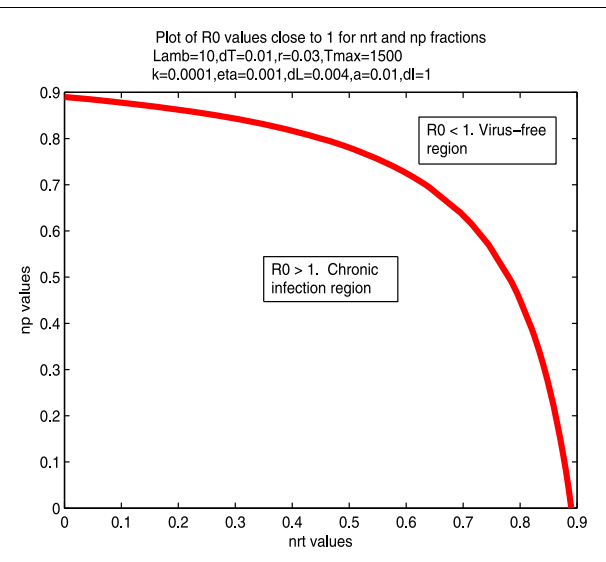

(b) Data set 2 .

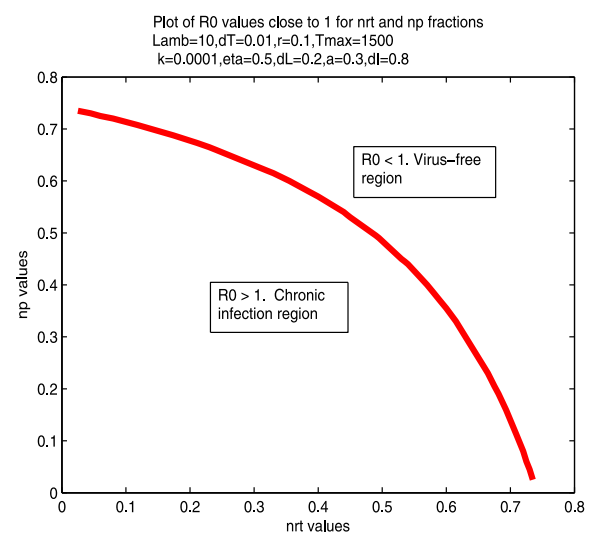

(c) Data set 3 .

Figure 1 Plots of the curve $R_{0}=1$ separating the virus-free and chronic equilibrium regions as a function of $n_{r t}$ and $n_{p}$ for the data sets 1,2 and 3 in Table 10.

\subsection{Sensitivity analysis}

For the sensitivity analysis, we consider three cases: (1) Virus-free equilibrium with $R_{0}<1$, (2) Chronic equilibrium with $R_{0} \approx 1$, and (3) Chronic equilibrium with $R_{0}>1$. 
Virus-free case. For this case, we use data set 1 in Table 10 and values of $n_{r t}=0.8, n_{p}=0.6$. For these parameter values, $R_{0}=0.47087<1$ and the equilibrium population values and eigenvalues are as follows:

$$
\begin{array}{ll}
\text { Virus-free } & \left(T_{1}^{*}, L_{1}^{*}, I_{1}^{*}, V_{1}^{*}, W_{1}^{*}\right)=(1177.4,0,0,0,0) . \\
\text { Eigenvalues } & -20,-0.0870,-0.9884,-0.5282,-20.474 . \\
\text { Chronic } & \left(T_{2}^{*}, L_{2}^{*}, I_{2}^{*}, V_{2}^{*}, W_{2}^{*}\right)=(2500.5,-45.9,-231.75,-4635,-6952.5) .
\end{array}
$$

Eigenvalues $\quad-20,-20.977,0.2124,-0.4030,-0.1043$.

As predicted for $R_{0}<1$, the virus-free equilibrium is locally asymptotically stable as the real parts of all eigenvalues are negative. Also, the chronic equilibrium does not exist because the infected T-cell and free virus populations are negative. It is also unstable because at least one eigenvalue has a positive real part.

The sensitivity indices for the virus-free healthy T-cell population $\left(T_{1}^{*}\right)$ are shown in Table 11 and for the basic reproductive number $R_{0}$ in Table 12 .

From the numerical results in Table 12, it can be seen that the most effective methods of reducing $R_{0}$ are the following: (1) to try to increase the efficacies $n_{r t}$ and $n_{p}$ of the antiretroviral therapy and (2) to increase virus clearance rate $c$, decrease infection rate $k$, or decrease viral reproduction rate $N$.

Chronic case $\left(R_{0} \approx 1\right)$. For this case, we use data set 1 in Table 10 and values of $n_{r t}=0.575$, $n_{p}=0.6$. For these parameter values, $R_{0}=1.0006 \approx 1$ and the equilibrium populations and eigenvalues are as follows:

$$
\begin{array}{ll}
\text { Virus-free } & \left(T_{1}^{*}, L_{1}^{*}, I_{1}^{*}, V_{1}^{*}, W_{1}^{*}\right)=(1177.4,0,0,0,0) . \\
\text { Eigenvalues } & -20,-0.0870,-0.1199,0.000475,-20.982 . \\
\text { Chronic } & \left(T_{2}^{*}, L_{2}^{*}, I_{2}^{*}, V_{2}^{*}, W_{2}^{*}\right)=(1176.7,0.0120,0.0605,1.210,1.815) . \\
\text { Eigenvalues } & -20,-20.981,-0.000477,-0.0867,-0.1198 .
\end{array}
$$

As predicted for $R_{0}>1$, the virus-free equilibrium is unstable as the real part of at least one eigenvalue is positive. Also, the chronic equilibrium exists because the infected T-cell

Table 11 Sensitivity indices for $T_{1}^{*}$

\begin{tabular}{llll}
\hline Parameter & Sensitivity index & Parameter & Sensitivity index \\
\hline$T_{\max }$ & 0.9024 & $\Lambda$ & 0.00976 \\
$r$ & 0.2472 & $d_{T}$ & -0.3449 \\
\hline
\end{tabular}

Table 12 Sensitivity indices for virus-free basic reproductive number $R_{0}$

\begin{tabular}{llll}
\hline Parameter & Sensitivity index & Parameter & Sensitivity index \\
\hline$T_{\max }$ & 0.9024 & $\Lambda$ & 0.00976 \\
$r$ & 0.2472 & $d_{T}$ & -0.3449 \\
$d_{L}$ & -0.000196 & $a$ & 0.00196 \\
$c$ & -1 & $k$ & 1 \\
$N$ & 1 & $n_{r t}$ & -4 \\
$n_{p}$ & -1.5 & $\eta$ & -0.000198 \\
\hline
\end{tabular}


Table 13 Sensitivity indices for $R_{0}$ and for chronic infected T-cells and virus populations for $R_{0} \approx 1$

\begin{tabular}{lllccc}
\hline Parameter & $\boldsymbol{R}_{\mathbf{0}}$ & $\mathbf{T}_{\mathbf{2}}^{*}$ & $\mathbf{L}_{\mathbf{2}}^{*}$ & $\mathbf{I}_{\mathbf{2}}^{*}$ & $\mathbf{V}_{\mathbf{2}}^{\boldsymbol{*}}$ \\
\hline$T_{\max }$ & 0.9024 & 0 & 1525.9 & 1525.9 & 1525.9 \\
$\Lambda$ & 0.00976 & 0 & 16.618 & 16.618 & 16.618 \\
$r$ & 0.2472 & 0 & 419.24 & 419.24 & 419.24 \\
$d_{T}$ & -0.3449 & 0 & -583.54 & -583.54 & -583.54 \\
$d_{\boldsymbol{L}}$ & -0.000196 & 0.000196 & -0.3414 & -0.332 & -0.33164 \\
$a$ & 0.00196 & -0.00196 & 2.3261 & 3.3164 & 3.3164 \\
$c$ & -1 & 1 & -1690.2 & -1690.2 & -1691.2 \\
$k$ & 1 & -1 & 1690.2 & 1690.2 & 1690.2 \\
$N$ & 1 & -1 & 1690.2 & 1690.2 & 1691.2 \\
$n_{r t}$ & -1.3529 & 1.3529 & -2286.7 & -2286.7 & -2286.7 \\
$n_{p}$ & -1.5 & 1.5 & -2535.3 & -2535.3 & -2536.8 \\
$\eta$ & -0.000198 & 0.000198 & -0.6652 & 0.6650 & -0.3350 \\
$d_{l}$ & 0 & 0 & 0 & -1 & 0 \\
\hline
\end{tabular}

and free virus populations are positive. It is also locally asymptotically stable because the real parts of all eigenvalues are negative. Also, since $R_{0} \approx 1$, the infected T-cell and free virus levels are close to zero and could easily be undetectable. The sensitivity indices for the chronic equilibrium with $R_{0} \approx 1$ are shown in Table 13 for $R_{0}$ and for the infected T-cell and virus populations $T_{2}^{*}, L_{2}^{*}, I_{2}^{*}$ and $V_{2}^{*}$.

Chronic case $\left(R_{0}>1\right)$. For this case, we use data set 1 in Table 10 and values of $n_{r t}=0.4$, $n_{p}=0.6$. For these parameter values, $R_{0}=1.4126>1$ and the equilibrium populations and eigenvalues are as follows:

$$
\begin{array}{ll}
\text { Virus-free } & \left(T_{1}^{*}, L_{1}^{*}, I_{1}^{*}, V_{1}^{*}, W_{1}^{*}\right)=(1177.4,0,0,0,0) . \\
\text { Eigenvalues } & -20,-0.0870,-0.1067,0.3657,-21.360 . \\
\text { Chronic } & \left(T_{2}^{*}, L_{2}^{*}, I_{2}^{*}, V_{2}^{*}, W_{2}^{*}\right)=(833.5,4.3624,22.026,440.52,660.78) . \\
\text { Eigenvalues } & -20,-20.981,-0.0405 \pm 0.1500 i,-0.1054 .
\end{array}
$$

As predicted for $R_{0}>1$, the virus-free equilibrium is unstable as the real part of at least one eigenvalue is positive. Also, the chronic equilibrium exists because the infected T-cell and free virus populations are positive. It is also locally asymptotically stable because the real parts of all eigenvalues are negative. The complex conjugate eigenvalue with the small negative real part indicates that the infected populations will oscillate with a slowly decreasing amplitude to the chronic equilibrium solution.

The sensitivity indices for the chronic equilibrium with $R_{0}>1$ are shown in Table 14 for $R_{0}$ and for the infected T-cell and virus populations $T_{2}^{*}, L_{2}^{*}, I_{2}^{*}$ and $V_{2}^{*}$.

From the numerical results in Tables 13 and 14, it can be seen that in the chronic infection region the most effective methods of reducing the free virus population $V_{2}^{*}$ are the following: (1) to try to increase the efficacies $n_{r t}$ and $n_{p}$ of the antiretroviral therapy and (2) to increase virus clearance rate $c$, decrease infection rate $k$, or decrease viral reproduction rate $N$.

\subsection{Dynamic behavior of solutions}

We used Matlab to integrate the system (1)-(5) for the parameter values in data set 1 in Table 10 and the $n_{r t}$ and $n_{p}$ values for the virus-free case $\left(n_{r t}=0.8, n_{p}=0.6\right)$ and for the 
Table 14 Sensitivity indices for chronic infected T-cells and virus populations for $R_{0}>1$

\begin{tabular}{llllll}
\hline Parameter & $\boldsymbol{R}_{\mathbf{0}}$ & $\boldsymbol{T}_{\mathbf{2}}^{*}$ & $\mathbf{L}_{\mathbf{2}}^{*}$ & $\mathbf{I}_{\mathbf{2}}^{*}$ & $\boldsymbol{V}_{\mathbf{2}}^{*}$ \\
\hline$T_{\max }$ & 0.9024 & 0 & 2.1023 & 2.1023 & 2.1023 \\
$\Lambda$ & 0.00976 & 0 & 0.1647 & 0.1647 & 0.1647 \\
$r$ & 0.2472 & 0 & 1.6811 & 1.6811 & 1.6811 \\
$d_{T}$ & -0.3449 & 0 & -1.1350 & -1.1350 & -1.1350 \\
$d_{L}$ & -0.000196 & 0.000196 & -0.01021 & -0.000501 & -0.000501 \\
$a$ & 0.00196 & -0.00196 & -0.9852 & 0.00501 & 0.00501 \\
$c$ & -1 & 1 & -1.5562 & -1.5562 & -2.5562 \\
$k$ & 1 & -1 & 1.5562 & 1.5562 & 1.5562 \\
$N$ & 1 & -1 & 1.5562 & 1.5562 & 2.5562 \\
$n_{r t}$ & -0.6667 & 0.6667 & -1.0375 & -1.0375 & -1.0375 \\
$n_{p}$ & -1.5 & 1.5 & -2.3344 & -2.3344 & -3.8344 \\
$\eta$ & -0.000198 & 0.000198 & 0.9997 & 0.9995 & -0.000506 \\
$d_{l}$ & 0 & 0 & 0 & -1 & 0 \\
\hline
\end{tabular}

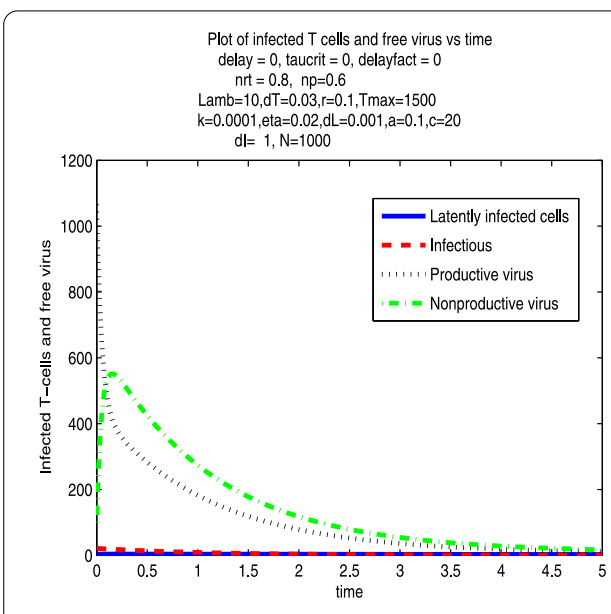

(a) Virus-free parameter values.

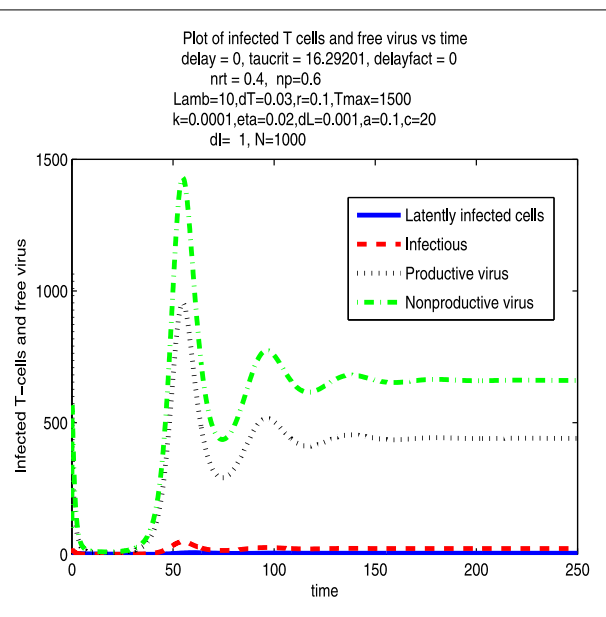

(b) Chronic parameter values.

Figure 2 Plots of populations vs time for virus-free and chronic parameter values.

chronic case with $R_{0}>1\left(n_{r t}=0.4, n_{p}=0.6\right)$. These parameter values correspond to cases 1 and 3 , respectively, discussed in the sensitivity analysis section.

Examples of the time-dependence of the solutions for zero time delay for infected T-cells and free virus are shown in Figure 2(a) for the virus-free case and (b) for the chronic case. The populations converge to the virus-free equilibrium $(1177.4,0,0,0,0)$ in Figure 2(a) and to the chronic equilibrium populations $(833.5,4.3624,22.026,440.52,660.78)$ in Figure 2(b). As noted for the chronic case with $R_{0}>1$ in the previous section, the solutions oscillate with decreasing amplitude due to the dominant complex conjugate pair of eigenvalues of the chronic equilibrium Jacobian.

\subsection{Andronov-Hopf bifurcation for chronic solutions}

One of the main conditions for the existence of an Andronov-Hopf bifurcation (see, e.g., [18]) is the existence of a purely imaginary pair of eigenvalues of the Jacobian of the chronic equilibrium at a critical value of a delay time with all other eigenvalues having negative real parts. In this paper, we find purely imaginary eigenvalues and the critical delay time by direct numerical solution of the characteristic equation $\operatorname{det}(\lambda I-J)=0$, where $J$ is the Jacobian in equation (12). 
Table 15 Examples of critical delay times $\tau_{c}$ (days) for Andronov-Hopf bifurcation

\begin{tabular}{|c|c|c|c|c|c|c|}
\hline & \multicolumn{2}{|l|}{ Set 1} & \multicolumn{2}{|l|}{ Set 2} & \multicolumn{2}{|l|}{ Set 3} \\
\hline & $\left(n_{r t}, n_{p}\right)$ & $\tau_{c}$ & $\left(n_{r t}, n_{p}\right)$ & $\tau_{c}$ & $\left(n_{r t}, n_{p}\right)$ & $\tau_{c}$ \\
\hline \multirow[t]{4}{*}{$R_{0}>1$} & $(0.2,0.3)$ & 15.429 & $(0.2,0.3)$ & 216.215 & $(0.2,0.3)$ & 14.171 \\
\hline & $(0.5,0.5)$ & 16.077 & $(0.5,0.5)$ & 216.299 & $(0.2,0.4)$ & 26.563 \\
\hline & $(0.4,0.6)$ & 16.292 & $(0.4,0.6)$ & 216.313 & $(0.25,0.4)$ & 44.064 \\
\hline & $(0.5,0.6)$ & 19.175 & $(0.5,0.6)$ & 216.405 & $(0.25,0.43)$ & 132.673 \\
\hline$R_{0} \approx 1$ & $(0.575,0.6)$ & 18.052 & $(0.633,0.7)$ & 231.147 & & \\
\hline
\end{tabular}

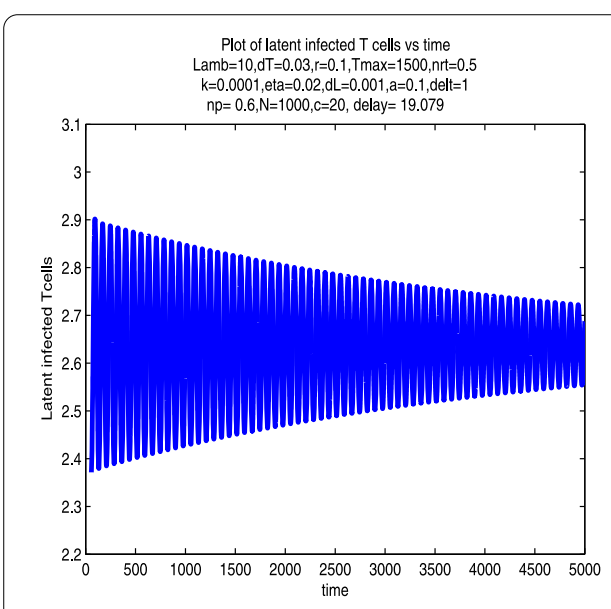

(a) Delay $\tau=0.995 \tau_{c}$.

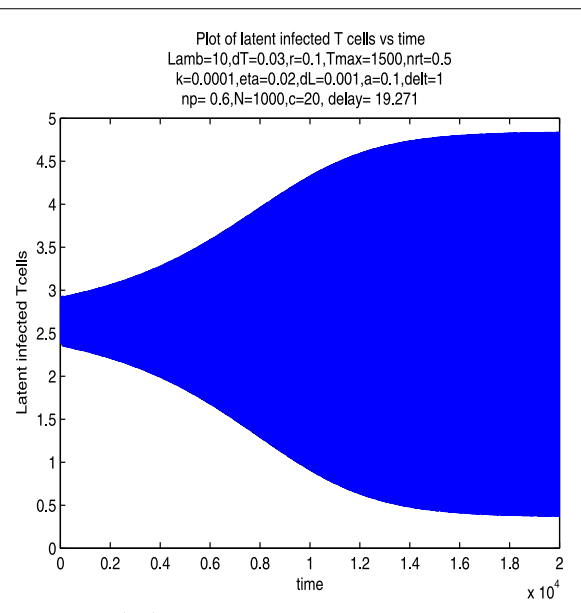

(b) Delay $\tau=1.005 \tau_{c}$.

Figure 3 Plots of latent T-Cell populations vs time for data set 1 for time delays less than and greater than critical time $\left(n_{r t}=0.5, n_{p}=0.6\right)$.

We have used Matlab to obtain numerical solutions of the characteristic equation $\operatorname{det}(\lambda I-J)=0$ for parameter values given in data sets 1,2 and 3 of Table 10 for a selection of $n_{r t}$ and $n_{p}$ values corresponding to $R_{0} \approx 1$ and $R_{0}>1$.

Examples of these critical values for data sets 1, 2 and 3 of Table 10 are shown in Table 15 . The critical delay values for a bifurcation are clearly very different for the three data sets. A noticeable difference between the data sets is also that the critical values for data sets 1 and 2 appear to change slowly as the level of the antiretroviral therapy is increased, whereas the critical values for data set 3 change rapidly as the level of the antiretroviral therapy is increased. The reason for this difference is at present unknown to the authors and requires a sensitivity analysis of the critical delay times.

An example of the dynamical behavior of the solutions of the model for data set 1 of Table 10 for the antiretroviral therapy levels $n_{r t}=0.5$ and $n_{p}=0.6$ are shown in Figure 3 for delay times just less than and just greater than the critical delay time. The plot for delay time just less than the critical time shows convergence to the chronic latent T-cell equilibrium population, whereas the plot for the delay time just greater than the critical time shows convergence to an oscillating population value. An example of the limit cycle behavior of the infectious free virus population for data set 1 in Table 10 for a time delay just greater than the critical delay is shown in Figure 4. The phase plane plot in Figure 4(b) shows a clear limit cycle behavior as predicted by Andronov-Hopf bifurcation theory (see, e.g., [18]). 


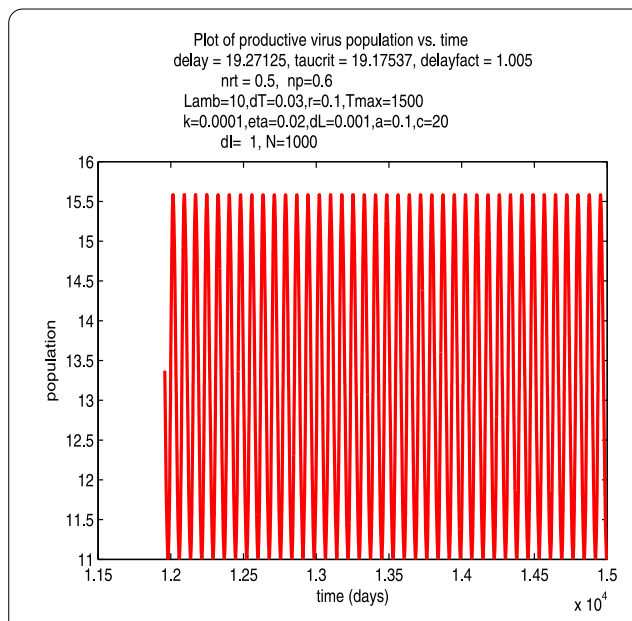

(a) Dynamical behavior.

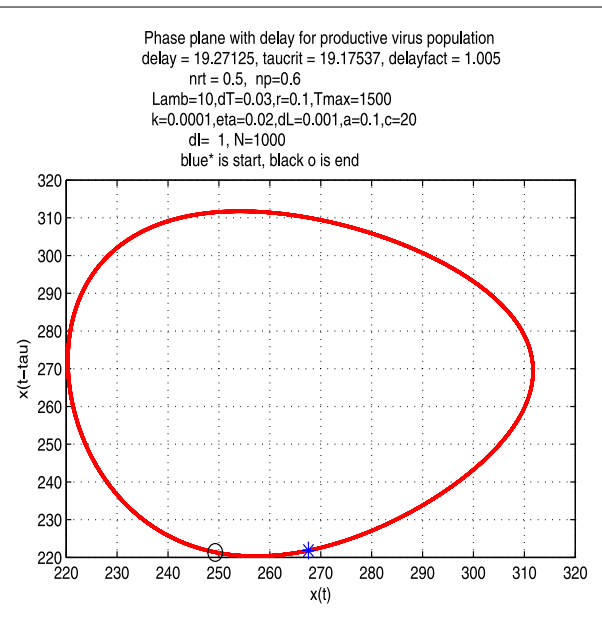

(b) Phase plane.

Figure 4 Limit cycle behavior of infectious free virus population for data set 1 for time delay greater than critical time for $n_{r t}=0.5, n_{p}=0.6$.

In numerical results, not shown in this paper, we have found that in the limit cycle region the model (1)-(5) predicts that the latently infected CD4+ T-cell population $L$ can become negative. Since this is physiologically impossible, it is necessary to put a lower bound on the $L$ population. The evidence (see, e.g., $[2,3]$ ) that the virus cannot be completely eliminated suggests that placing a positive lower bound on $L$ would give a more realistic model.

\section{Conclusion}

We have obtained simple analytical formulas for the sensitivity indices of this time-delay HIV model and used them to compute numerical values. We have found the following:

(1) For the virus-free equilibrium, the virus-free healthy T-cell population depends on parameters that cannot be changed easily and the chronic healthy T-cell population is only useful because it is used to compute the sensitivity indices for $R_{0}$.

A reduction in $R_{0}$ is important because it corresponds to a faster convergence of the infected populations to zero. From the numerical results, it can be seen that the most effective methods of reducing $R_{0}$ are the following: (1) to try to increase the efficacies $n_{r t}$ and $n_{p}$ of the antiretroviral therapy and then (2) to increase virus clearance rate $c$, decrease infection rate $k$, or decrease viral reproduction rate $N$.

(2) For the chronic equilibrium, reduction of the productively infected viral population $V_{2}^{*}$ is the most important method of reducing the HIV infection. From the numerical results, the most effective methods of reducing $V_{2}^{*}$ are the same as for the virus-free case.

(3) The numerical results show that Andronov-Hopf bifurcations occur in the time delay model and that the critical delay times can vary over a wide range. For three data sets published by [15] and selected from the work of previous authors (Table 10), we have found delay times ranging from approximately 15-20 days to more than 200 days.

As stated in the introduction, one aim of examining the effect of introducing a time delay for procession of latently infected CD4+ T-cells to productively infected T-cells was to check if Andronov-Hopf bifurcations could produce limit cycle behavior in the free 
virus populations that might be associated with the intermittent viral blips with period of approximately 50 days observed by Rong and Perelson $[1,5,6]$. Our results show that Andronov-Hopf bifurcations associated with this time delay in procession can produce limit cycle behavior with periods similar to the viral blip period. However, the present authors are not able to claim that this behavior actually causes the viral blips.

In numerical results, not shown in this paper, we have found that for a range of antiretroviral levels and delay times the model (1)-(5) predicts that the latently infected CD4+ T-cell population $L$ can become negative. However, in these cases, all other populations remain positive. The evidence (see, e.g., $[2,3])$ that the virus cannot be completely eliminated suggests that placing a positive lower bound on $L$ is necessary to obtain a more realistic time-delay model.

Competing interests

The authors declare that they have no competing interests.

\section{Authors' contributions}

All authors contributed equally to the writing of this paper. All authors read and approved the final manuscript.

\section{Author details}

${ }^{1}$ Faculty of Science, Energy and Environment, King Mongkuts University of Technology North Bangkok (Rayong Campus), Rayong, Thailand. 'Department of Mathematics, King Mongkuts University of Technology North Bangkok, Bangkok, Thailand. ${ }^{3}$ Centre of Excellence in Mathematics, CHE, Si Ayutthaya Road, Bangkok 10400, Thailand.

\section{Publisher's Note}

Springer Nature remains neutral with regard to jurisdictional claims in published maps and institutional affiliations.

Received: 31 January 2017 Accepted: 1 May 2017 Published online: 16 May 2017

\section{References}

1. Rong, LB, Perelson, AS: Modeling latently infected cell activation: viral and latent reservoir persistence, and viral blips in HIV-infected patients on potent therapy. PLoS Comput. Biol. 5(10), 1-18 (2009)

2. Chomont, N, El-Far, M, Ancuta, P, Trautman, L, Procopio, FA, Yassine-Diab, B, Boucher, G, Boulasse, M-R, Ghattas, G, Brenchley, JM, Schacker, TW, Hill, BJ, Douek, DC, Routy, J-P, Haddad, EK, Sékaly, R-P: HIV reservoir size and persistence are driven by T cell survival and homeostatic proliferation. Nat. Med. 15(8), 893-900 (2009)

3. AIDS.gov: https://www.aids.gov. Accessed 30 April 2017

4. Callaway, DS, Perelson, AS: HIV-1 infection and low steady state viral loads. Bull. Math. Biol. 64(1), 29-64 (2002)

5. Rong, LB, Perelson, AS: Asymmetric division of activated latently infected cells may explain the decay kinetics of the HIV-1 latent reservoir and intermittent viral blips. Math. Biosci. 217(1), 77-87 (2009)

6. Rong, LB, Perelson, AS: Modeling HIV persistence, the latent reservoir, and viral blips. J. Theor. Biol. 260(2), 308-331 (2009)

7. Wilson, C: A farewell to condoms. New Scientist., 22-23 (11 February 2017)

8. Unaids: (2016) http://www.unaids.org/sites/default/files/media_asset/global-AIDS-update2016_en.pdf. Accessed 31 May 2016

9. World Health Organization: (2017) http://www.who.int/campaigns/tb-day/2017/en/. Accessed 24 March 2017

10. Banks, HT, Davidiana, M, Shuhua, H, Kepler, GM, Rosenberg, ES: Modeling HIV immune response and validation with clinical data. J. Biol. Dyn. 2(4), 357-385 (2008)

11. Barton, KM, Burch, BD, Soriano-Sarabia, N, Margolis, DM: Prospects for treatment of latent HIV. Clin. Pharmacol. Ther. 93(1), 46-56 (2013)

12. Wang, LC, Li, MY: Mathematical analysis of the global dynamics of a model for HIV infection of CD4 ${ }^{+}$T cells. Math. Biosci. 200(1), 44-57 (2006)

13. Wang, Y, Zhou, YC, Wu, JH, Heffernan, J: Oscillatory viral dynamics in a delayed HIV pathogenesis model. Math. Biosci. 219(2), 104-112 (2009)

14. Wang, Y, Zhou, Y, Brauer, F, Huffernan, JM: Viral dynamics model with CTL immune response incorporating antiretroviral therapy. J. Math. Biol. 67(4), 901-934 (2013)

15. Wang, Y, Lui, J, Lui, L: Viral dynamics of an HIV model with latent infection incorporating antiretroviral therapy. Adv. Differ. Equ. 2016, 225 (2016)

16. Ding, $Y, X u, M, H u, L$ : Asymptotic behavior and stability of a stochastic model for AIDS transmission. Appl. Math. Comput. 204, 99-108 (2008)

17. Robert, MG, Saha, AK: The asymptotic behavior of logistic epidemic model with stochastic disease transmission. Appl. Math. Lett. 12, 37-41 (1999)

18. Kuznetsov, YA: Elements of Applied Bifurcation Theory, 3rd edn. Springer, New York (2004)

19. Luenberger, DG: Introduction to Dynamic Systems: Theory, Models and Applications. Wiley, New York (1979)

20. Vanden Driessche, $P$, Watmough, J: Reproduction numbers and sub-threshold endemic equilibria for compartmental models of disease transmission. Math. Biosci. 180(1), 29-48 (2002) 
21. Chitnis, N, Hyman, JM, Cushing, JM: Determining important parameters in the spread of malaria through the sensitivity analysis of a mathematical model. Bull. Math. Biol. 70, 1272-1296 (2008)

22. de Leenheer, P, Smith, HL: Virus dynamics: a global analysis. SIAM J. Appl. Math. 63(4), 1313-1327 (2003)

Submit your manuscript to a SpringerOpen ${ }^{\circ}$ journal and benefit from:

- Convenient online submission

- Rigorous peer review

- Immediate publication on acceptance

- Open access: articles freely available online

- High visibility within the field

- Retaining the copyright to your article

Submit your next manuscript at $\gg$ springeropen.com 